\title{
Optical coherence tomography classification of diabetic cystoid macular edema
}

This article was published in the following Dove Press journal:

Clinical Ophthalmology

27 August 2013

Number of times this article has been viewed

\author{
Yasser M Helmy \\ Heba R Atta Allah \\ Department of Ophthalmology, Minia \\ University, Minya, Egypt
}

Purpose: To propose a new classification of diabetic cystoid macular edema (CME) based on optical coherence tomography (OCT) findings and cover all new important findings.

Patients and methods: A retrospective study was carried out in the El-Minia Investigation Eye Center between January 2012 and November 2012. It included 104 eyes of 86 patients, aged between 50 and 71 years, all with type II diabetes mellitus of duration from 5 to 20 years. All patients were diagnosed to have CME, as assessed by OCT, with measurement of the vertical size of the largest macular cyst and maximum macular thickness, and the relation between them.

Results: Patients were divided into four groups. Eyes with cysts less than $30 \%$ of macular thickness were considered to have CME I $(n=4,3.84 \%)$, while those between $30 \%$ and $60 \%$ were considered to have CME II $(n=62,59.62 \%)$. Eyes with cysts between $60 \%$ and $90 \%$ of macular thickness were considered to have CME III $(n=36,34.62 \%)$. CME IV was diagnosed when the size of the cyst became more than $90 \%$ of the macular thickness, and this was encountered in two eyes $(1.92 \%)$.

Conclusions: OCT is a useful technique for quantitative measurement and helps in better anatomical characterization of CME, and this classification of diabetic CME may be of value in classification of CME due to causes other than diabetes.

Keywords: optical coherence tomography, cystoid macular edema, diabetic retinopathy

\section{Introduction}

Diabetic retinopathy is the leading cause of blindness in working populations in industrially developed countries. With the rising incidence of diabetes mellitus, this ocular disease will be an ever greater worldwide health issue in the near future than it is already today. ${ }^{1}$

Approximately $25 \%$ of people with diabetes have at least some form of diabetic retinopathy, and the incidence increases with the duration of the diabetes. Diabetic macular edema increases with the duration of diabetes, and the prevalence is $5 \%$ within the first 5 years after diagnosis and $15 \%$ at 15 years. ${ }^{2}$

Diabetic macular edema (DME) is the main cause of visual loss in diabetic patients. The term "macular edema" refers to the actual retinal thickening in the region of the macula, secondary to a rupture of the inner hematoretinal barrier. The diagnosis of macular edema is based on binocular slit-lamp biomicroscopy, leakage on fluorescein angiography (FA), and recently on the qualitative and quantitative information on retinal structure and thickness offered by optical coherence tomography (OCT). ${ }^{3}$
Correspondence: Heba Radi Atta Allah Department of Ophthalmology, Minia University, 32 Adnan El-Maleky Street, Minya 6IIII, Egypt

Tel +20 I00 2554107

Email radi_heba@yahoo.com 
With the advent of OCT, several investigators have classified DME on the basis of the retinal map and cross-sectional appearance of the retina on OCT. ${ }^{4}$ Spectral domain (SD) OCT allows for better characterization of the retinal morphology. The morphological patterns of DME on OCT are generally classified into diffuse retinal thickening, cystoid macular edema (CME), subretinal detachment, and vitreomacular interface abnormalities. ${ }^{5}$

CME is a cause of severe visual loss that occurs in a variety of pathologic conditions, such as age-related macular degeneration, diabetic retinopathy, branch or central retinal vein occlusion, epiretinal membrane or vitreomacular traction, and as a complication of intraocular surgery. ${ }^{6} \mathrm{CME}$ is a pathologic definition with two components: abnormal collection of extracellular fluid and cystoid-space formation. ${ }^{7}$

OCT, first described by Huang et $\mathrm{al}^{8}$ in 1991, is an imaging modality capable of providing high-resolution cross-sectional images of the neurosensory retina. As OCT is noninvasive, it was quickly adopted by clinicians for the assessment of patients with CME. In part, because of the relative ease with which they can detect CME, many clinicians now prefer OCT over FA for its detection. ${ }^{9}$

OCT has gained increasing popularity as an objective tool to measure retinal thickness and other aspects associated with macular edema. ${ }^{10}$ Standard OCT assessment of DME has been adopted in multicenter trials in patients with diabetic retinopathy by the Diabetic Retinopathy Clinical Research Network. An advantage of using OCT is its quantitative assessment, rather than the qualitative evaluation performed with photography or biomicroscopy. ${ }^{11}$

A lot of information that is available now by OCT demonstrates that diabetic CME is a complex entity rather than a simple clinical definition. We therefore propose a new classification of diabetic cystoid macular edema based on OCT findings to better cover all new important findings.

\section{Patients and methods}

The study was done retrospectively in the El-Minia Investigation Eye Center between January 2012 and November 2012. The study included 104 eyes of 86 patients. The age of the patients ranged between 50 and 71 years, and all had type II diabetes mellitus of duration from 5 and 20 years. All patients were diagnosed to have CME, as assessed by OCT examination.

Patients with CME due to any cause other than diabetes mellitus were excluded from the study, eg, pseudophakic, vascular occlusion, any macular traction other than diabetic causes, history of topical medications, or with history of any intraocular surgery or trauma. Also, all patients who had had previous laser photocoagulation were excluded from the study.

All patients were subjected to:

- ophthalmic and medical history-taking

- slit-lamp examination of the anterior segment

- dilated fundus examination using slit-lamp biomicroscopy with 78 D lens (Nidek Slit Lamp SL-450; Nidek, Co., Ltd., Japan)

- OCT examination using the Cirrus HD-OCT model 4000 (Zeiss, Jena, Germany) with the following technique:

- after appropriate pupillary dilatation using cyclopentolate $1 \%$ eye drops, the patient was seated comfortably on a chair in front of the device, in a dimly lit room, asked to place their chin on the chin rest, and to look at the center of the green target, and not at the moving light inside the imaging aperture

- selecting scan type - after identifying a patient and clicking acquire, the acquire screen appears; we used two scan types for each eye:

$>$ macular cube $512 \times 128$ - this generates a cube of data through a $6 \mathrm{~mm}^{2}$ grid by acquiring a series of 128 horizontal scan lines, each composed of 512 A-scans, except for the central high-definition vertical and horizontal scans, which are composed of 1,024 A-scans; this scan helps in giving an idea about the regions of maximum height, so they are better assessed and scanned by the high-definition 5-line raster. Also, we can get a radial line printout (six B-scan areas extracted at the meridians of $0^{\circ}$, $30^{\circ}, 60^{\circ}, 90^{\circ}, 120^{\circ}$, and $150^{\circ}$; the B-scan is used to produce a two-dimensional, cross-sectional view of the eye and the orbit)

high-definition five-line raster - this scan gives the highest resolution of all the Cirrus scan types; it scans through five parallel lines of equal length, and each line is composed of 4,096 A-scans (in this study, $6 \mathrm{~mm}$ line length vertically, horizontally and obliquely oriented at $45^{\circ}$ and $135^{\circ}$ ); it was selected to evaluate the mean retinal thickness from the innermost internal limiting membrane to the retinal pigment epithelium at the fovea (radius $500 \mu \mathrm{m}$ ); the central fovea was defined as the location without the inner retinal layers in the macular region.

Multiple scans were taken throughout the whole macular area. Scans with the strongest signal strength were selected (signal strength of more than 6/10). According to these scans, we were able to measure certain dimensions and evaluate certain morphological changes and findings within the scans. 
We measured the vertical dimensions of the macular cysts, and the maximum retinal thickness. We defined cystoid spaces on OCT B-scans as circular or ovoid intraretinal hyporeflective spaces present at the same approximate transverse location on two adjacent B-scans.

Any disruption of the external limiting membrane (ELM) was searched for within the central $1 \mathrm{~mm}$ of the fovea. If the ELM line appeared to be complete at the fovea in all scans, we diagnosed it as an intact ELM. Any discontinuity or interruption of the ELM line in one scan or more was considered a disrupted ELM layer.

We identified the integrity of the inner segment/outer segment (IS/OS) line beneath the fovea using the same criteria described for the ELM line. If the line appeared to be complete at the fovea in all scans, it was diagnosed as an intact IS/OS line. If there was an incomplete IS/OS line in one scan or more, it was considered a disrupted IS/OS layer.

The presence of hyper-reflective foci in the outer retinal layers from the ELM to the retinal pigment epithelium within the $1 \mathrm{~mm}$ scanned area centered on the fovea was given a plus sign (+ was added if the patient had hyper-reflective foci, as previously described). Other findings, looked for during the scan analysis, included neurosensory detachment and vitreoretinal traction.

We classified patients into four groups based on the ratio of vertical size of the largest macular cyst in relation to the size of maximum macular thickness, with the use of OCT. Patients with cysts less than (30\%) of macular thickness were considered to have CME I, while those between $30 \%$ and $60 \%$ of macular thickness were considered to have CME II. Patients with cysts between $60 \%$ and $90 \%$ of macular thickness were considered to have CME III. CME IV was diagnosed when the size of the cyst became more than $90 \%$ of the macular thickness.

Each grade was then subdivided into (A, B, C, and D), according to certain morphological findings, and other associations:

- A - cysts without any disruption to the ELM or IS/OS junction lines

- $\mathrm{B}$ - cysts with ELM disruption

- $\mathrm{C}$ - cysts with IS/OS disruption

- D - cysts with disruption of both ELM and IS/OS junction lines.

The study adhered to the tenets of the Declaration of Helsinki.

\section{Statistical analysis}

The statistical program SPSS for Windows version 11 (IBM, Armonk, NY, USA) was used for data entry and analysis.
Quantitative data were presented as means and standard deviation, while qualitative data were presented as frequency distributions.

\section{Results}

The study was done retrospectively at the El-Minia Investigation Eye Center between January 2012 and November 2012. The study included 104 eyes of 86 patients. The age of the patients ranged between 50 and 71 years (mean age $61.2 \pm 6.1$ years), and all had type II diabetes mellitus for between 5 and 20 years (range $12.1 \pm 4$ years). All patients were diagnosed with CME, as assessed by OCT examination.

We classified patients into four groups based on the ratio of vertical size of the largest macular cyst in relation to the size of maximum macular thickness measured using OCT. Eyes with cysts less than $30 \%$ of macular thickness were considered to have CME I, present in four cases (3.84\%), while those between $30 \%$ and $60 \%$ were considered to have CME II, present in 62 eyes (59.62\%). Eyes with cysts between $60 \%$ and $90 \%$ of macular thickness were considered to have CME III, present in 36 eyes (34.62\%). CME IV was diagnosed when the size of the cyst became more than $90 \%$ of the macular thickness, and was encountered in two eyes $(1.92 \%)$ (Table 1).

Accordingly, statistical analysis of the cases revealed:

- CME I - four eyes (3.84\%) had cysts less than 30\% of macular thickness; all had intact ELM, but with IS/ OS disruption (inner/outer photoreceptor segments [CME I C]), as seen in Table 2;

- CME II - 62 eyes (59.62\%) had cysts between 30\% and $60 \%$ of macular thickness; 18 eyes $(17.3 \%)$ had intact ELM and IS/OS junction lines (CME II A) (Figure 1), 14 eyes (13.46\%) had IS/OS disruption (CME II C), and four eyes (3.84\%) had IS/OS disruption with hyperreflective foci $\left(\mathrm{CME}\right.$ II $\left.\mathrm{C}^{+}\right)$; 12 eyes $(11.52 \%)$ had disruption in ELM and IS/OS junction lines (CME II D), and 14 eyes (13.46\%) had disruption in ELM and IS/ OS junction lines with hyper-reflective foci (CME II $\left.\mathrm{D}^{+}\right)$ (Figure 2); no eyes were detected with ELM disruption alone (CME II B), as seen in Table 3;

Table I Frequency of eyes in each grade of CME

\begin{tabular}{lll}
\hline Grade & Number of eyes & Percentage \\
\hline CME I & 4 & $3.84 \%$ \\
CME II & 62 & $59.62 \%$ \\
CME III & 36 & $34.62 \%$ \\
CME IV & 2 & $1.92 \%$ \\
\hline
\end{tabular}

Abbreviation: CME, cystoid macular edema. 
Table 2 Distribution of eyes with CME I

\begin{tabular}{lll}
\hline CME I & $\mathbf{n}$ & $\%$ \\
& $\mathbf{4}$ & $\mathbf{3 . 8 4}$ \\
\hline $\begin{array}{l}\text { A. Cysts less than 30\%, without any disruption } \\
\text { of ELM or IS/OS }\end{array}$ & 0 & 0 \\
+ Hyper-reflective foci presence & 0 & 0 \\
B. Cysts with ELM disruption & 0 & 0 \\
+ Hyper-reflective foci presence & 0 & 0 \\
C. Cysts with IS/OS disruption & 4 & 3.84 \\
$\quad+$ Hyper-reflective foci presence & 0 & 0 \\
D. Cysts with disruption of both ELM and IS/OS & 0 & 0 \\
junction lines & & \\
$\quad+$ Hyper-reflective foci presence & 0 & 0
\end{tabular}

Abbreviations: CME, cystoid macular edema; ELM, external limiting membrane; IS/ OS, inner segment/outer segment.

- CME III - 36 eyes (34.62\%) had cysts between $60 \%$ and $90 \%$ of macular thickness, six eyes $(5.76 \%)$ had intact ELM and IS/OS junction lines (CME III A), four eyes (3.84\%) had ELM disruption (CME III B), and four eyes (3.84\%) had IS/OS disruption (CME III C); six eyes $(5.76 \%)$ had disruption in ELM and IS/OS junction lines (CME III D) (Figure 3), and 16 eyes (15.38\%) had disruption in ELM and IS/OS junction lines with hyper-reflective foci $\left(\mathrm{CME}_{\mathrm{III} \mathrm{D}}{ }^{+}\right)$, as seen in Table 4;

- CME IV - impending rupture cysts or cysts $>90 \%$ of macular thickness were present in two eyes $(1.92 \%)$ with both ELM and IS/OS disruption (CME IV D) (Figure 4).

Other associated findings including neurosensory detachment and vitreoretinal traction (either by epiretinal membranes or partially detached posterior hyaloid) were present, as seen in Table 5.

Regarding CME I and CME IV, no associated findings were detected, as seen in Table 5. In eyes with CME II, 24 eyes $(23 \%)$ had neurosensory detachment (Figure 2 ), eight

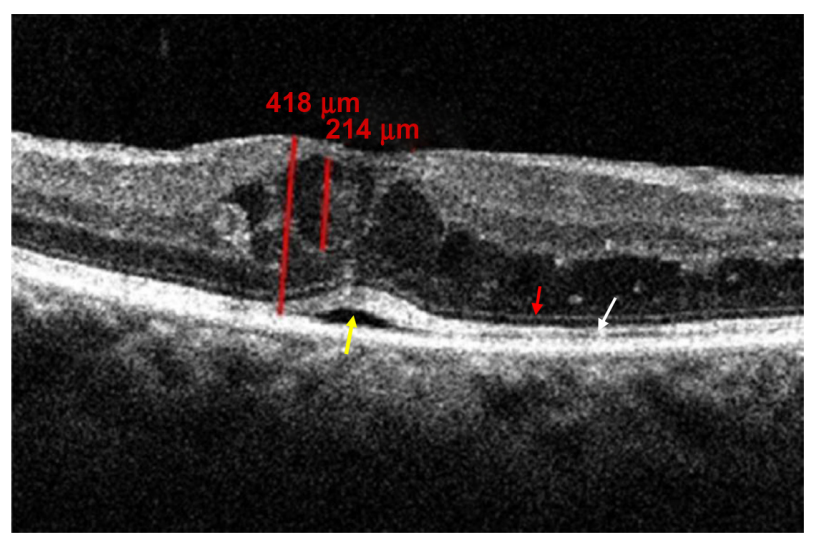

Figure I OCT scan showing CME II A with intact ELM (red arrow) and IS/OS junction line (white arrow), with shallow neurosensory detachment (yellow arrow). Abbreviations: CME, cystoid macular edema; OCT, optical coherence tomography; ELM, external limiting membrane; IS/OS, inner segment/outer segment.

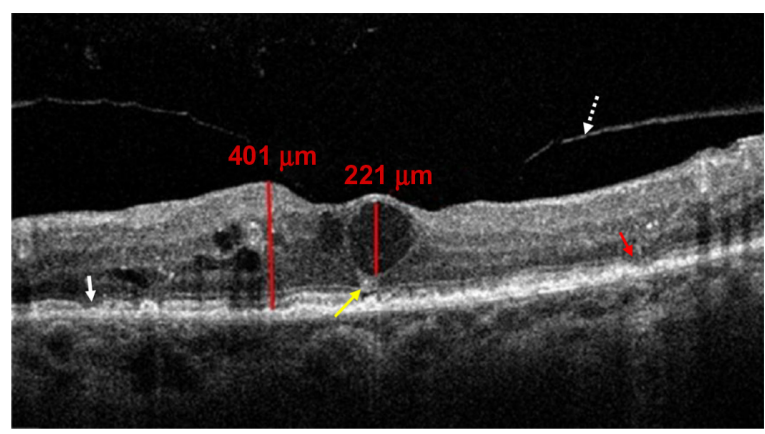

Figure 2 OCT scan showing CME II $D^{+}$with disruption of ELM (red arrow) and IS/ OS junction line (white arrow). Hyper-reflective foci (yellow arrow) and partial PVD without traction (white dotted arrow) are also shown.

Abbreviations: CME, cystoid macular edema; OCT, optical coherence tomography; ELM, external limiting membrane; IS/OS, inner segment/outer segment; PVD, posterior vitreous detachment.

eyes $(7.68 \%)$ had epiretinal membranes with tangential vitreoretinal traction, and four eyes $(3.84 \%)$ had partially detached posterior hyaloids without traction (Figure 3). In CME III, 18 eyes (17.3\%) had neurosensory detachment, and 12 eyes $(11.53 \%)$ had epiretinal membranes with tangential vitreoretinal traction (Figure 3 ).

\section{Discussion}

DME is one of the main causes of visual impairment in patients with diabetic retinopathy. ${ }^{3} \mathrm{CME}$ is a pathologic definition with two components: abnormal collection of extracellular fluid and cystoid-space formation. ${ }^{7}$

The common diagnostic tools for assessing macular edema are stereo-ophthalmoscopy and FA. However, these methods are subjective, and seem to be insensitive to small changes in retinal thickness. ${ }^{12}$

OCT provides detailed information about retinal microstructure, and measures retinal thickness with high precision and reproducibility. The recently introduced SD OCT machines have numerous improvements that enhance our

Table 3 Distribution of eyes with CME II

\begin{tabular}{lll}
\hline CME II & $\mathbf{n}$ & $\%$ \\
& $\mathbf{6 2}$ & $\mathbf{5 9 . 6 2}$ \\
\hline $\begin{array}{l}\text { A. Cysts between 30\% and 60\%, without any } \\
\text { disruption to the ELM or IS/OS junction lines }\end{array}$ & 18 & 17.3 \\
$\quad+$ Hyper-reflective foci presence & 0 & 0 \\
B. Cysts with ELM disruption & 0 & 0 \\
+ Hyper-reflective foci presence & 0 & 0 \\
C. Cysts with IS/OS disruption & 14 & 13.46 \\
$\quad+$ Hyper-reflective foci presence & 4 & 3.84 \\
D. Cysts with disruption of both ELM and IS/OS & 12 & 11.52 \\
junction lines & & \\
$\quad+$ Hyper-reflective foci presence & 14 & 13.46 \\
\hline
\end{tabular}

Abbreviation: CME, cystoid macular edema; ELM, external limiting membrane; IS/ OS, inner segment/outer segment. 


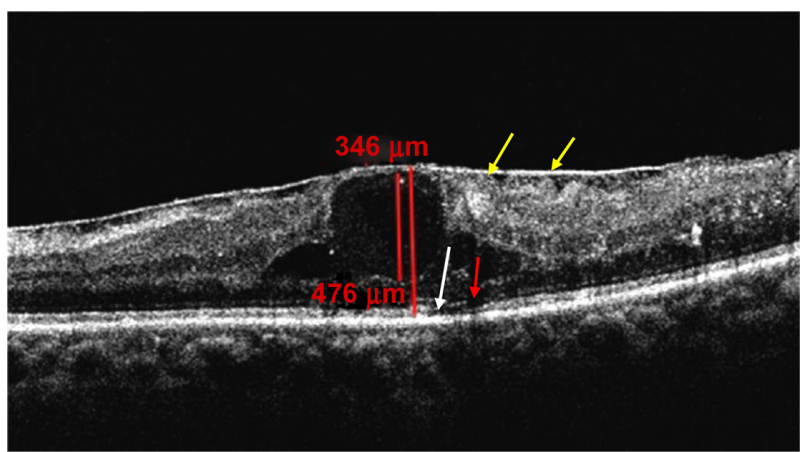

Figure 3 OCT showing CME III D with disruption of ELM (red arrow) and IS/OS junction line (white arrow). ERM and areas of tangential vitreoretinal traction (yellow arrows) are also shown.

Abbreviations: CME, cystoid macular edema; OCT, optical coherence tomography; ELM, external limiting membrane; IS/OS, inner segment/outer segment; ERM, epiretinal membrane.

ability to examine retinal microstructure and obtain more reliable measurements. ${ }^{13}$

Any abnormal pooling of extracellular fluid may result in displacement of the spatial relationships between retinal neuronal components. Small amounts of fluid may lead to an increase in overall retinal thickness, whereas larger amounts may give rise to cell-free spaces, as seen in CME. On OCT images, cystoid cavities appear as black non-reflective spaces, surrounded by medium-to-low reflective septa. ${ }^{14}$

Ozdek et al found that $40 \%$ of CMEs detected on OCT were not detected by biomicroscopy, and 63\% were not detected even on FA. OCT is thus a better diagnostic tool to diagnose $\mathrm{CME}$ in patients with diabetic retinopathy than biomicroscopy or FA. ${ }^{15}$

Hee and coworkers demonstrated that the central macular thickness measured by OCT correlates with visual acuity with a sensitivity greater than that of slit-lamp biomicroscopy. In addition, they commented that even in the absence of fluorescein leakage, there could be increases in retinal thickness and loss of visual acuity. ${ }^{10}$

Table 4 Distribution of eyes with CME III

\begin{tabular}{lll}
\hline CME III & $\mathbf{n}$ & $\%$ \\
& 36 & $\mathbf{3 4 . 6 2}$ \\
\hline A. Cysts between 60\% and 90\%, without any & 6 & 5.76 \\
$\begin{array}{l}\text { disruption to the ELM or IS/OS junction lines } \\
\text { + Hyper-reflective foci presence }\end{array}$ & 0 & 0 \\
B. Cysts with ELM disruption & 4 & 3.84 \\
+ Hyper-reflective foci presence & 0 & 0 \\
C. Cysts with IS/OS disruption & 4 & 3.84 \\
$\quad+$ Hyper-reflective foci presence & 0 & 0 \\
D. Cysts with disruption of both ELM and IS/OS & 6 & 5.76 \\
$\begin{array}{l}\text { junction lines } \\
\text { + Hyper-reflective foci presence }\end{array}$ & 16 & 15.38 \\
\hline
\end{tabular}

Abbreviations: CME, cystoid macular edema; ELM, external limiting membrane; IS/OS, inner segment/outer segment.

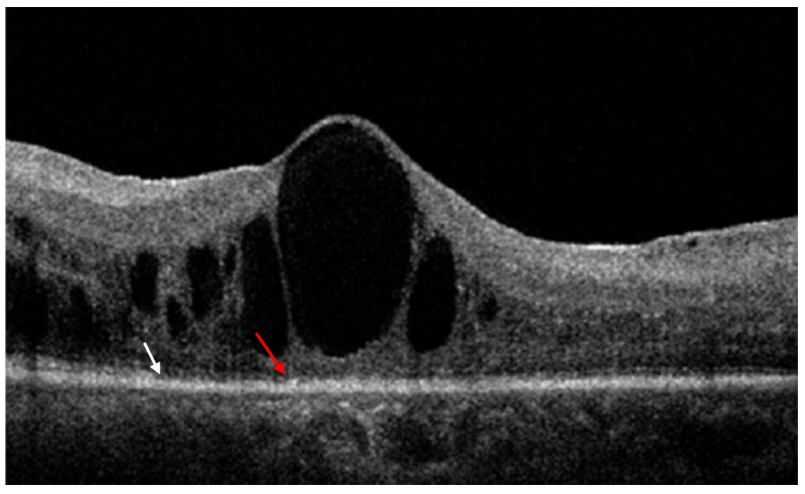

Figure 4 OCT scan showing CME IV D. Large impending rupture cyst with disruption of ELM (red arrow) and IS/OS junction line (white arrow).

Abbreviations: $\mathrm{CME}$, cystoid macular edema; OCT, optical coherence tomography; ELM, external limiting membrane; IS/OS, inner segment/outer segment.

Several studies have found a modest correlation between OCT-measured retinal thickness and visual acuity. Central retinal thickness has a more significant effect on visual acuity than does age, fluorescein leakage, hemoglobin $A_{1 c}$, perifoveal capillary blood-flow velocity, or severity of perifoveal capillary occlusion. ${ }^{16,17}$

To our knowledge, there have been limited trials to classify diabetic CME on an OCT basis. Panozzo et al classified CME into mild, intermediate, and severe grades according to the size of cysts. ${ }^{18}$ Also, Koleva-Georgieva and Sivkova subdivided cystoid DME into mild, moderate, and severe according to the size of cystoid spaces. Mild cystoid DME presents with small cysts mainly in the outer retinal layers. The cystoid spaces in eyes with intermediate and severe cystoid DME were mainly located in the outer layers, predominantly in the fovea. ${ }^{19}$

These previous studies emphasized the importance of determining the size of the cysts, which determine to a large extent the degree of visual acuity of these patients. Also, these studies considered that large confluent cystoid cavities might involve almost the entire thickness of the retina and give a retinoschisis appearance.

In our study, we categorized these data more precisely depending upon the relative vertical size of the largest cyst in relation to the maximum thickness of the macula. We assumed that fluid accumulation in cystoid spaces determines progression of macular edema along three stages of the disease. At the fourth stage, disruption of cystic septa

Table 5 Associated findings with CME

\begin{tabular}{|c|c|c|c|c|}
\hline & CME I & CME II & CME III & CME IV \\
\hline $\begin{array}{l}\text { Neurosensory } \\
\text { detachment }\end{array}$ & 0 & $24(23 \%)$ & I8 (I7.3\%) & 0 \\
\hline Epiretinal membrane & 0 & $8(7.68 \%)$ & $12(11.52 \%)$ & 0 \\
\hline $\begin{array}{l}\text { Partially detached } \\
\text { posterior hyaloid }\end{array}$ & 0 & $4(3.84 \%)$ & 0 & 0 \\
\hline
\end{tabular}

Abbreviation: CME, cystoid macular edema. 
occurs, resulting in formation of a large confluent cyst of more than $90 \%$ of the macular thickness.

OCT clearly delineates the ELM, which corresponds to the adherens junctions between the Müller cells and photoreceptor cells and also prevents passage of macromolecules. The IS/OS junction on OCT images might represent photoreceptor function, which is light perception. These layers on OCT images reportedly provide important information about pathologies of the photoreceptors. ${ }^{20}$

OCT has been used to assess the status of the photoreceptor layer. A close relationship of this status with visual function has been reported in various diseases, such as branch retinal vein occlusion, central serous chorioretinopathy, retinitis pigmentosa, and retinal detachment. Further, an association has been detected between visual acuity and abnormal OCT findings, such as those pertaining to the ELM and the IS/OS junction. ${ }^{21}$

Since the introduction of SD OCT, it has become possible to visualize accurately the outer retinal layers and define the integrity of the photoreceptor IS/OS junction and the ELM layer. The integrity of these layers has been reported to correlate with retinal function, and discussion in the literature is ongoing about its prognostic value. Several authors have found out that visual acuity has a positive correlation with the survival rate of ELM and IS/ $\mathrm{OS},{ }^{22}$ and that the postoperative status of the photoreceptors is related to the final visual function after resolution of normal retinal morphology following surgery for persistent $\mathrm{DME}^{21}$ or epiretinal membrane. ${ }^{23}$ The percentage disruption of the photoreceptor IS/OS junction layer is a significant predictor of visual acuity. ${ }^{24}$ Analysis of the integrity of IS/ OS and ELM on SD OCT scans should be a part of CME evaluation.

Because of the importance of the integrity of the ELM and IS/OS layers as prognostic features of visual improvement after treatment for DME, inclusion of these important data was involved in our classification of diabetic CME. Presence of disruption of either ELM or IS/OS alone or combined was included in our subdivision of grades of CME.

One of the drawbacks of our study is that we could not give a percentage to the integrity of the ELM and IS/OS layers in our classification, as assumed by some authors. Assessing the status of the IS/OS junction on OCT helps clinicians more accurately predict the visual outcome of patients with diabetic CME.

Fine lesions (hyper-reflective foci) have been newly delineated on OCT images, and the presence of these hyperreflective foci in the outer retina is closely associated with a disrupted ELM and IS/OS lines and decreased visual acuity (VA) in DME. ${ }^{25}$

Small hyper-reflective foci have been identified in patients with DME. These foci can be less than $30 \mathrm{~mm}$ in diameter and cannot be identified on fundus biomicroscopy, FA, or infrared imaging. They were characterized by the same amount of hyper-reflectivity as the accumulated dots in an area of hard exudates, thus they may represent tiny intraretinal protein and/or lipid deposits acting as precursors of hard exudates. ${ }^{26}$

Hyper-reflective foci, which may be precursors of hard exudates, were associated significantly with heterogeneity or higher levels of reflectivity in cystoid spaces on the OCT images. In other words, the foci were observed in the cystoid spaces with severe disruption of the blood-retinal barrier (BRB). Although the origin of the hyper-reflective foci remains controversial, condensed proteins or lipids in the cystoid spaces might deposit as hyper-reflective foci, resulting in emergence of hard exudates. ${ }^{27}$ Another possibility is that lipid-laden macrophages might take up the concentrated proteins or lipids in the cystoid spaces. A severe break in the vascular walls might allow macrophage migration into the cystoid spaces, regardless of whether the BRB breakdown is or is not induced by inflammatory cells. ${ }^{28}$ The accumulation of hyper-reflective foci in the subretinal spaces indicates a poor prognosis in DME. ${ }^{27}$

Inclusion of hyper-reflective foci in our classification of diabetic CME adds beneficial and worthwhile information reflecting the degree of $\mathrm{BRB}$ breakdown and its impact on the definitive visual acuity of patients.

This study was limited by a small sample size and its retrospective nature. The duration of edema for each patient at the time of the SD OCT examination was variable. This mixture of patients did provide a broad overview of the spectrum of diabetic CME, and was more representative of the variability of clinical presentation.

This classification of diabetic CME may be of value in the classification of CME due to causes other than diabetes. OCT is a useful technique for quantitative measurement and helps in better anatomical characterization of CME, and is thereby more relevant while planning management strategies, follow-up, explaining prognosis and predicting visual outcome.

\section{Acknowledgments}

Our thanks and gratefulness to all members of the El-Minia Investigation Eye Center.

\section{Disclosure}

The authors report no conflicts of interest in this work. 


\section{References}

1. Klein R, Klein BEK, Moss SE, Cruickshanks KJ. The Wisconsin Epidemiologic Study of Diabetic Retinopathy: XVII. The 14-year incidence and progression of diabetic retinopathy and associated risk factors in type 1 diabetes. Ophthalmology. 1998;105:1801-1815.

2. Aiello LP, Gardner TW, King GL, et al. Diabetic retinopathy. Diabetes Care. 1998;21:143-156.

3. Brown JC, Solomon SD, Bressler SB, Schachat AP, DiBernardo C, Bressler NM. Detection of diabetic foveal edema. Contact lens biomicroscopy compared with optical coherence tomography. Arch Ophthalmol. 2004;122:330-335.

4. Kim NR, Kim YJ, Chin HS, Moon YS. Optical coherence tomographic patterns in diabetic macular oedema: prediction of visual outcome after focal laser photocoagulation. Br J Ophthalmol. 2009;93: 901-905.

5. Otani T, Kishi S, Maruyama Y. Patterns of diabetic macular oedema with optical coherence tomography. Am J Ophthalmol. 1999;127:88-93.

6. Johnson MW. Etiology and treatment of macular edema. Am J Ophthalmol. 2009;147:11-21.

7. Tso MO. Pathology of cystoid macular edema. Ophthalmology. 1982;89:902-915.

8. Huang D, Swanson EA, Lin CP, et al. Optical coherence tomography. Science. 1991;254:1178-1181.

9. Kozak I, Morrison VL, Clark TM, et al. Discrepancy between fluorescein angiography and optical coherence tomography in detection of macular disease. Retina. 2008;28:538-544.

10. Hee MR, Puliafito CA, Wong C, et al. Quantitative assessment of macular edema with optical coherence tomography. Arch Ophthalmol. 1995;113:1019-1029.

11. Diabetic Retinopathy Clinical Research Network [website on the Internet]. Available from: http://www.drcr.net. Accessed June 27, 2013.

12. Hee MR, Puliafito CA, Duker JS, et al. Topography of diabetic macular edema with optical coherence tomography. Ophthalmology. 1998;105:360-370.

13. Polito A, Del Borrello M, Isola M, Zemella N, Bandello F. Repeatability and reproducibility of fast macular thickness mapping using Stratus optical coherence tomography. Arch Ophthalmol. 2005;123:1330-1337.

14. Antcliff R, Marshall J. The pathogenesis of edema in diabetic maculopathy. Semin Ophthalmol. 1999;14:223-232.

15. Ozdek SC, Erdinç MA, Gürelik G, Aydin B, Bahçeci U, Hasanreisoğlu B. Optical coherence tomographic assessment of diabetic macular edema: comparison with fluorescein angiographic and clinical findings. Ophthalmologica. 2005;219:86-92.
16. Network DR, Browning DJ, Glassman AR, et al. Relationship between optical coherence tomography-measured central retinal thickness and visual acuity in diabetic macular edema. Ophthalmology. 2007;114: 525-536.

17. Darrell EB. Optical coherence tomography in diabetic macular edema. Curr Opin Ophthalmol. 2010;21:172-177.

18. Panozzo G, Parolini B, Gusson E, et al. Diabetic macular edema: an OCT-based classification. Semin Ophthalmol. 2004;19:13-20.

19. Koleva-Georgieva DN, Sivkova NP. Types of diabetic macular edema assessed by optical coherence tomography. Folia Med. 2008;50: 30-38.

20. Marmor MF. Mechanisms of fluid accumulation in retinal edema. Doc Ophthalmol. 1999;97:239-249.

21. Sakamoto A, Nishijima K, Kita M, Oh H, Tsujikawa A, Yoshimura N. Association between foveal photoreceptor status and visual acuity after resolution of diabetic macular edema by pars plana vitrectomy. Graefes Arch Clin Exp Ophthalmol. 2009;247:1325-1330.

22. Otani T, Yamaguchi Y, Kishi S. Correlation between visual acuity and foveal microstructural changes in diabetic macular edema. Retina. 2010;30:774-780.

23. Mitamura Y, Hirano K, Baba T, Yamamoto S. Correlation of visual recovery to presence of photoreceptor inner/outer segment junction in optical coherence images after epiretinal membrane surgery. $\mathrm{Br} J$ Ophthalmol. 2009;93:171-175.

24. Maheshwary AS, Oster SF, Yuson RM, Cheng L, Mojana F, Freeman WR. The association between percent disruption of the photoreceptor inner segment/outer segment and visual acuity in diabetic macular edema. Am J Ophthalmol. 2010;150:63-67.

25. Uji A, Murakami T, Nishijima K, et al. Association between hyper reflective foci in the outer retina, status of photoreceptor layer, and visual acuity in diabetic macular edema. Am J Ophthalmol. 2012;153: $710-717$.

26. Bolz M. Optical coherence tomographic hyper reflective foci: a morphologic sign of lipid extravasation in diabetic macular edema. Ophthalmology. 2009;116:914-920.

27. Ota M, Nishijima K, Sakamoto A, et al. Optical coherence tomographic evaluation of foveal hard exudates in patients with diabetic maculopathy accompanying macular detachment. Ophthalmology. 2010;117: 1996-2002.

28. Murakami T, Nishijima K, Sakamoto A, et al. Association of pathomorphology, photoreceptor status, and retinal thickness with visual acuity in diabetic retinopathy. Am J Ophthalmol. 2011;151:310-317.
Clinical Ophthalmology

\section{Publish your work in this journal}

Clinical Ophthalmology is an international, peer-reviewed journal covering all subspecialties within ophthalmology. Key topics include: Optometry; Visual science; Pharmacology and drug therapy in eye diseases; Basic Sciences; Primary and Secondary eye care; Patient Safety and Quality of Care Improvements. This journal is indexed on Submit your manuscript here: http://www.dovepress.com/clinical-ophthalmology-journal

\section{Dovepress}

PubMed Central and CAS, and is the official journal of The Society of Clinical Ophthalmology (SCO). The manuscript management system is completely online and includes a very quick and fair peer-review system, which is all easy to use. Visit http://www.dovepress.com/ testimonials.php to read real quotes from published authors. 